\title{
Malabsorption syndrome associated with carcinoma of the bronchus
}

\author{
A. G. WANGEL AND D. J. DELLER \\ From the Department of Medicine, University of Adelaide, South Australia
}

EDITORIAL SYNOPSIS This paper describes an association between bronchial carcinoma and steatorrhoea. The histology of the jejunum was abnormal. The mucosal changes might be due to the systemic effects of a neoplasm.

It is well known that some of the manifestations of carcinoma of the bronchus cannot be explained by the local effects of the malignancy or by distant metastases. The systemic manifestations of malignancy are of importance because they may occur when the lesion in the lung is clinically occult and their study may provide knowledge concerning the biological nature of malignancy itself. Recently a number of metabolic abnormalities have been described: Cushing's syndrome (Kovach and Kyle, 1958); hypercalcaemia (Connor, Thomas, and Howard, 1956); the carcinoid syndrome (Williams and Azzopardi, 1960); and the syndrome of hyponatraemia and persistent renal sodium loss (Schwartz Bennett, Curelop, and Bartter, 1957). Turner and Williams (1962) have reported a case with steatorrhoea as well as hyponatraemia. The tumour was of the oat-cell variety, as appears to be the case with most bronchial carcinomas possessing endocrine activity (McDaniel, Pittman, Hill, and Starnes, 1963). We now report a further case demonstrating the association of steatorrhoea and bronchial carcinoma. In our case the steatorrhoea was gross, other features of the malabsorption syndrome were present, sodium metabolism was not affected, and the tumour was a squamous carcinoma. Attempts were made during life and at necropsy to define the cause of the steatorrhoea.

\section{CASE REPORT}

A male retired tramways worker, aged 71 , became aware of increasing pain and stiffness of the wrists, ankles, and knees in August 1961. He smoked 10 cigarettes a day and had an unproductive cough. On examination a fortnight after the arthritic symptoms had begun, there was clubbing of the fingers and toes, and swelling of the interphalangeal joints, wrists, and ankles. Radiographs of the joints showed hypertrophic osteo- arthropathy and of the chest patchy consolidation at both lung bases. No malignant cells were detected in the sputum. A blood count was normal but the sedimentation rate was $44 \mathrm{~mm}$. in the first hour (Westergren). At bronchoscopy the bronchus to the left lower lobe was narrowed just below its origin. A biopsy of this region was taken but no malignant tissue was obtained. The joint symptoms, which had become disabling, responded dramatically to treatment with phenylbutazone. This drug was continued for a further three months.

In October 1961 a rounded opacity appeared in the left hilum causing partial collapse of the left lung. A course of radiotherapy was given to the hilar region of the chest over a period of six weeks, when $5,400 \mathrm{r}$. was administered by means of a linear-accelerator. During treatment the left lung completely collapsed but re-expanded with physiotherapy. A deep vein thrombosis developed in the right leg but this subsided after a short course of phenindione. The patient remained in a satisfactory clinical condition for another eight months, requiring only a codeine linctus.

In August 1962 flatulence and diarrhoea commenced. The patient had three to six bowel actions daily and occasionally the urgency of defaecation resulted in incontinence. The motions were pale, bulky, offensive, and difficult to flush away. There was no abdominal pain. There was no previous history of diarrhoea, abdominal pain, or gastrointestinal surgery. The presence of steatorrhoea was confirmed by chemical methods: the faecal excretion of fat was $18 \mathrm{~g}$. per day (normal less than $5 \mathrm{~g}$. per day). Radiographs of the small intestine showed a widespread deficiency pattern without any localized abnormality. The patient was admitted to the Royal Adelaide Hospital in November 1962 for investigation of the steatorrhoea.

INVESTIGATIONS The investigations are summarized in Table I. Noteworthy features are the mild anaemia, which was macrocytic; the low serum level of folic acid activity; the impaired absorption of radioactive vitamin $B_{12}$ which was not corrected by the administration of intrinsic factor; the steatorrhoea; the low serum albumin level 
TABLE I

\section{INVESTIGATIONS ON ADMISSION}

${ }^{1}$ Haemoglobin
${ }^{1}$ Mean cell volume
Serum vitamin $B_{12}$
${ }^{1}$ Serum folic acid
${ }^{1} C^{58}$ vitamin $B_{12}$ alone
(Schilling test)

${ }^{1} C^{58}$-vitamin $B_{12}$ with Intrinsic factor
(Schilling test)

1 Faecal fat

d-xylose excretion

Serum albumin

$\mathrm{Cr}^{51}$-albumin excretion

IInsulin stimulation

${ }^{1}$ Augmented histamine test

Serum sodium

'Serum potassium

Serum chloride

Serum bicarbonate

Urinary sodium

Urinary potassium

Urinary 5-hydroxy indoleacetic acid

Serum calcium

Serum phosphate

'Serum alkaline phosphatase

Blood urea nitrogen

'Abnormal values obtained

but without evidence of abnormal gastrointestinal protein loss; and the low serum potassium level. An insulin stimulation test was combined with an augmented histamine test to ascertain the integrity of the vagi in gastric secretion, but these manoeuvres did not furnish this information because of the histamine-fast achlorhydria. The serum sodium level was normal and the urinary excretion of sodium was not raised. There was no detectable urinary excretion of 5-hydroxyindoleacetic acid or of other tryptophan derivatives. Small bowel biopsy and duodenal intubation for pancreatic function studies were attempted without success.

FAECAL FAT EXCRETION The effects of pancreatic supplements, cholinergic stimulation, and intestinal sterilization on the faecal fat excretion were successively tried (Table II). Pancreatic supplements were given as Pancrex V (pancreatin) 2 g. q.i.d. with meals for two weeks. Mecothane (carbaminoylbeta-methylcholine chloride)

TABLE II

FACTORS INFLUENCING THE STEATORRHOEA

\begin{tabular}{rlll}
$\begin{array}{l}\text { Experiment } \\
\text { No. }\end{array}$ & Date & Material Administered & $\begin{array}{l}\text { Faecal Fat } \\
\text { Excretion } \\
(\text { g./daty) }\end{array}$ \\
\hline 1 & 17.8 .62 & & $18 \cdot 0$ \\
2 & 17.11 .62 & - & $27 \cdot 7$ \\
3 & 27.11 .62 & Pancrex V & $12 \cdot 0$ \\
4 & 4.12 .62 & Pancrex V & $97 \cdot 5$ \\
5 & 10.12 .62 & Mecothane & $11 \cdot 1$ \\
6 & 17.12 .62 & Chlortetracycline & 8.0 \\
7 & 6.1 .63 & Vitamin supplements & $25 \cdot 0$ \\
8 & 14.1 .63 & Vitamin supplements & $21 \cdot 7$ \\
9 & 30.4 .63 & Vitamin supplements & 7.0 \\
10 & 26.6 .63 & Vitamin supplements & $27 \cdot 5$ \\
11 & 23.9 .63 & Vitamin supplements & $1 \cdot 9$
\end{tabular}

was administered for one week in a dosage of $25 \mathrm{mg}$. t.d.s., and $500 \mathrm{mg}$. q.i.d. chlortetracycline was given for a week. Faecal fat was estimated on the pooled three-day specimens at the end of each week. Another five estimations were made over a period of nine months when the patient was only receiving supplements of iron, folic acid, vitamin $B_{12}$, and potassium. None of these measures resulted in a clear-cut reduction in the diarrhoea or chemical steatorrhoea. In September 1963 the patient became constipated and excretion of faecal fat fell to normal; this did not appear to be related to any change in his therapeutic regime.

PROGRESS The diarrhoea and steatorrhoea lasted from August 1962 to September 1963. He then became constipated and his clinical condition deteriorated. He died from respiratory insufficiency on 4 January 1964. Within 15 minutes of death 11 . of formalin-saline solution was instilled through a gastric tube into the stomach and proximal intestine.

NECROPSY (Dr. R. T. W. Reid) The left pleural cavity was obliterated by dense fibrous adhesions. The right pleural cavity was dry without adhesions. Coronal sections through the left lung showed that much of the upper lobe contained cystic cavities with surrounding consolidation infiltrated by malignant tissue. The lower lobe was firm, collapsed, and diffusely infiltrated by necrotic malignant tissue which surrounded the main lower lobe bronchus. Some mediastinal lymph glands containing tumour had involved the posterior wall of the pericardium. The left vagus nerve was surrounded and

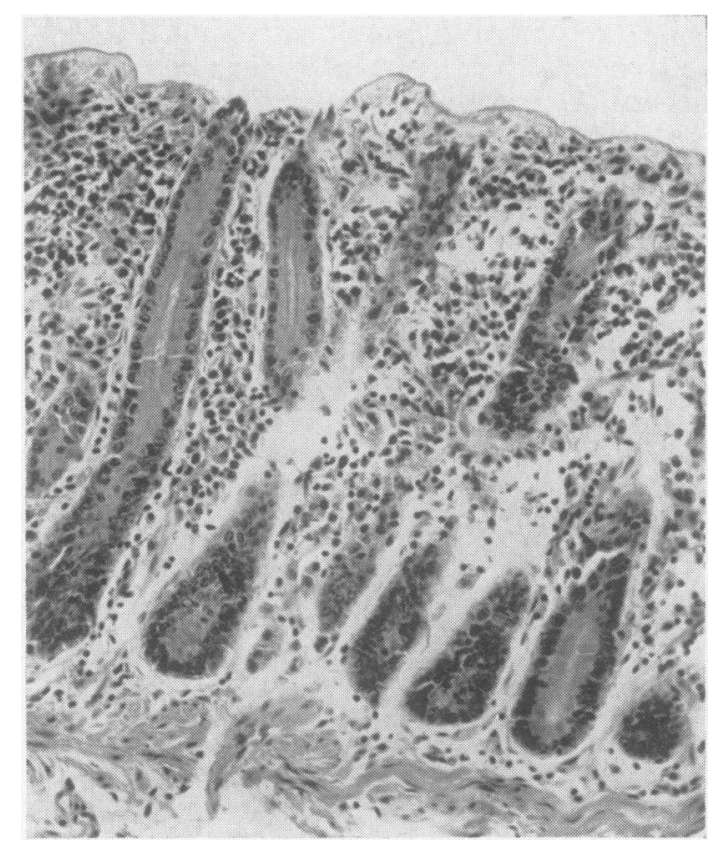

FIG. 1. Subtotal villous atrophy of the upper jejunum (× 140). 
compressed by malignant tissue where it curved under the aorta.

The stomach, duodenum, and upper $10 \mathrm{~cm}$. of jejunum were macroscopically well preserved and there was no malignant infiltration of the gut or mesenteric lymph nodes. The pancreas was normal and the adrenals were not remarkable. Small metastatic nodules were present in the lower poles of both kidneys. The visceral pericardium was coated by fibrinous exudate but the valves showed no abnormality. The brain was macroscopically normal.

The pulmonary tumour was a typical squamous cell carcinoma of bronchial origin. The stomach showed mild atrophic gastritis. There was patchy subtotal villous atrophy of the duodenum and upper jejunum (Fig. 1). These changes were not considered to be the result of post-mortem autolysis.

\section{DISCUSSION}

Carcinoma of the bronchus is an increasingly common disease accounting for $2 \%$ of all deaths in Australia in 1962 (Commonwealth Bureau of Statistics). Accordingly it is not unexpected that other diseases will be found in association with malignant chest disease. However, some of the disorders described as systemic manifestations of carcinoma of the bronchus are otherwise notably rare. Moreover a partial or complete remission has been reported following treatment of the primary lung tumour in many of them: in hypertrophic pulmonary osteoarthropathy (Vogl, Blumenfeld, and Gutner, 1955); in the neuromuscular syndromes (Brain and Henson, 1958); in hypercalcaemia (Connor et al., 1956); in gynaecomastia (Hardy, 1960); and in the syndrome of hyponatraemia and renal sodium loss (Schwartz et al., 1957). This patient initially presented with severe joint pains. The arthritic symptoms disappeared even before treatment with radiotherapy was given to the lesion in the chest. Diarrhoea and steatorrhoea commenced 12 months after the chest and joint symptoms and lasted 13 months. Then, quite suddenly the patient became constipated and the excretion of faecal fat returned to normal. There were no alimentary symptoms during the last four months of life. At necropsy none of the recognized causes of malabsorption in a man of his age were found: the pancreas did not show changes of chronic inflammation or tumour; there were no diverticuli or blind loops in the small intestine; none of the characteristic features of Whipple's disease were present; and there was no evidence of occlusion of the mesenteric vessels.

There are many possible causes of steatorrhoea in patients with carcinoma of the bronchus. Williams and Azzopardi (1960) reported a case of the malignant carcinoid syndrome due to oat-cell carcinoma of the bronchus in which the tumour contained 5-hydroxytryptamine. Although steatorrhoea was not a feature of this case, a defect in the absorption of fat has been reported in association with 5-H.T.-secreting tumours in other sites (Kowlessar, Law, and Sleisenger, 1959). However, there was no raised 5-hydroxyindole excretion to suggest the malignant carcinoid syndrome in the present case. Malabsorption secondary to extensive mucosal involvement of the small intestine by lymphatic blockage was excluded by the necropsy findings. Similarly there was no histological evidence of amyloidosis which occasionally results from carcinoma (Adlersberg and Schein, 1947). Interruption of the vagus due to compression of the nerve in the mediastinum is another possible explanation. However, in our patient only one division of the vagus nerve was involved in the malignant process and it is most unlikely that this would result in steatorrhoea. Steatorrhoea has also been reported following treatment with the anticoagulant drug phenindione (Juel-Jensen, 1959), the antibiotic neomycin (Faloon, Fisher, and Duggan, 1958), and the purgative phenolphthalein (French, Gaddie, and Smith, 1956). Phenindione had been given for a short period eight months before the onset of diarrhoea. Neither of the other drugs were administered. We have recently studied. a patient without carcinoma of the bronchus in whom steatorrhoea and features of the malabsorption syndrome appeared to follow prolonged treatment with phenylbutazone. However, in the present case there was an interval of six months between phenylbutazone being stopped and the onset of alimentary symptoms.

Occlusion of the superior mesenteric artery may be followed by steatorrhoea and other evidence of malabsorption for a number of months (Joske, Shamma'a, and Drummey, 1958). The cause of vascular occlusion in carcinoma of the bronchus could be either embolism from non-bacterial verrucal endocarditis or the hypercoagulable state that exists in carcinoma. Temporary occlusion of the mesenteric artery would account for the spontaneous remission of steatorrhoea that was observed in this patient. There were no findings, however, at necropsy to suggest that this condition had previously existed. Furthermore the patient had not complained of abdominal pain before the commencement of diarrhoea.

Diarrhoea and steatorrhoea are also wellrecognized complications of diabetes mellitus, often in association with diabetic neuropathy. It has been suggested that steatorrhoea is secondary to the neurological change affecting the small intestine (Rundles, 1945). In carcinoma of the bronchus a 
variety of neurological syndromes have been defined (Brain and Henson, 1958) and it is possible that an autonomic neuropathy may sometimes exist in this condition.

We have no positive evidence that the relationship of malabsorption and malignant disease is not merely coincidental. The possibility, however, remains that the malabsorption syndrome may be one of the systemic manifestations of carcinoma of the bronchus. There was an area of subtotal villous atrophy in the proximal jejunum at necropsy and this may have been more extensive during life, because steatorrhoea had ceased abruptly four months before death. In coeliac disease, where extensive subtotal villous atrophy of the mucosa is found, contact of gliadin or one of its component polypeptides and the mucosa of the small intestine results in malabsorption in susceptible individuals. Perhaps a polypeptide, which has a noxious influence on the intestine, is elaborated by some malignancies. The effect of a gluten-free diet on absorption was not tested in the present case, and this aspect should be studied in future cases.

\section{SUMMARY}

A patient with squamous-cell carcinoma of the bronchus and pulmonary osteoarthropathy developed steatorrhoea and other features of the malabsorption syndrome. Diarrhoea and steatorrhoea commenced 12 months after the chest and arthritic symptoms began and persisted for 13 months. There was a spontaneous remission of the steatorrhoea four months before death occurred. Investigations showed a mild macrocytic anaemia, subnormal level of folic acid activity, impaired absorption of radioactive vitamin $\mathbf{B}_{12}$ not corrected by intrinsic factor, hypoalbuminaemia, and hypokalaemia. The steatorrhoea was uninfluenced by pancreatic supplements, cholinergic stimulation, intestinal sterilization with antibiotics, or vitamin supplements. No recognized cause for steatorrhoea was revealed at necropsy although the proximal jejunum showed patchy villous atrophy and it is suggested that the malabsorption syndrome may represent another systemic effect of malignancy.

\section{REFERENCES}

Adlersberg, D., and Schein, J. (1947). Clinical and pathologic studies in sprue. J. Amer. med. Ass., 134, 1459-1467.

Brain, Sir R., and Henson, R. A. (1958). Neurological syndromes associated with carcinoma: the carcinomatous neuromyopathies. Lancet, 2, 971-975.

Connor, T. B., Thomas, W. C. Jr., and Howard, J. E. (1956). The etiology of hypercalcemia associated with lung carcinoma. J. clin. Invest., 35, 697-698.

Faloon, W. W., Fisher, C. J., and Duggan, K. C. (1958). Occurrence of a sprue-like syndrome during neomycin therapy. Ibid., 37, 893.

French, J. M., Gaddie, R., and Smith, N. M. (1956). Tropical sprue. A study of seven cases and their response to combined chemotherapy. Quart. J. Med., 25, 333-351.

Hardy, J. D. (1960). Gynecomastia associated with lung cancer. $J$. Amer. med. Ass., 173, 1462-1465.

Joske, R. A., Shamma'a, M. H., and Drummey, G. D. (1958). Intestinal malabsorption following temporary occlusion of the superior mesenteric artery. Amer. J. Med., 25, 449-455.

Juel-Jensen, B. E. (1959). Sensitivity to phenindione: report of a case with severe diarrhoea. Brit. med. J., 2, 173-174.

Kovach, R. D., and Kyle, L. H. (1958). Cushing's syndrome and bronchogenic carcinoma. Amer. J. Med., 24, 981-988.

Kowlessar, O. D., Law, D. H., and Sleisenger, M. H. (1959). Malabsorption syndrome associated with metastatic carcinoid tumor. Ibid. 27, 673-677.

McDaniel, H. G., Pittman, J. A., Hill, S. R., and Starnes, W. R. (1963). Adrenal cortical hyperfunction associated with bronchogenic carcinoma. Ibid. 35, 427-433.

Rundles, R. W. (1945). Diabetic neuropathy. General review with report of 125 cases. Medicine (Baltimore), 24, 111-160.

Schwartz, W. B., Bennett, W., Curelop, S., and Bartter, F. C. (1957). A syndrome of renal sodium loss and hyponatremia probably resulting from inappropriate secretion of antidiuretic hormone. Amer. J. Med., 23, 529-542.

Turner, P., and Williams, R. (1962). Unexplained steatorrhoea in the syndrome of hyponatraemia and carcinoma of bronchus. Brit. med. J., 1, 287-290.

Vogl, A., Blumenfeld, S., and Gutner, L. B. (1955). Diagnostic significance of pulmonary hypertrophic osteoarthropathy. Amer. J. Med., 18, 51-65.

Williams, E. D., and Azzopardi, J. G. (1960). Tumours of the lung and the carcinoid syndrome. Thorax, 15, 30-36. 